Document downloaded from:

http://hdl.handle.net/10251/64358

This paper must be cited as:

Martín Furones, AE.; Anquela Julián, AB.; Padin Devesa, J.; Berné Valero, JL. (2011). On Standard Reductions to Relative Gravity Measurements. A Case Study Through the Establishment of the New Local Gravity Net in the Province of Valencia (Spain). Survey Review. 43(319):16-29. doi:10.1179/003962610X12747001420825.

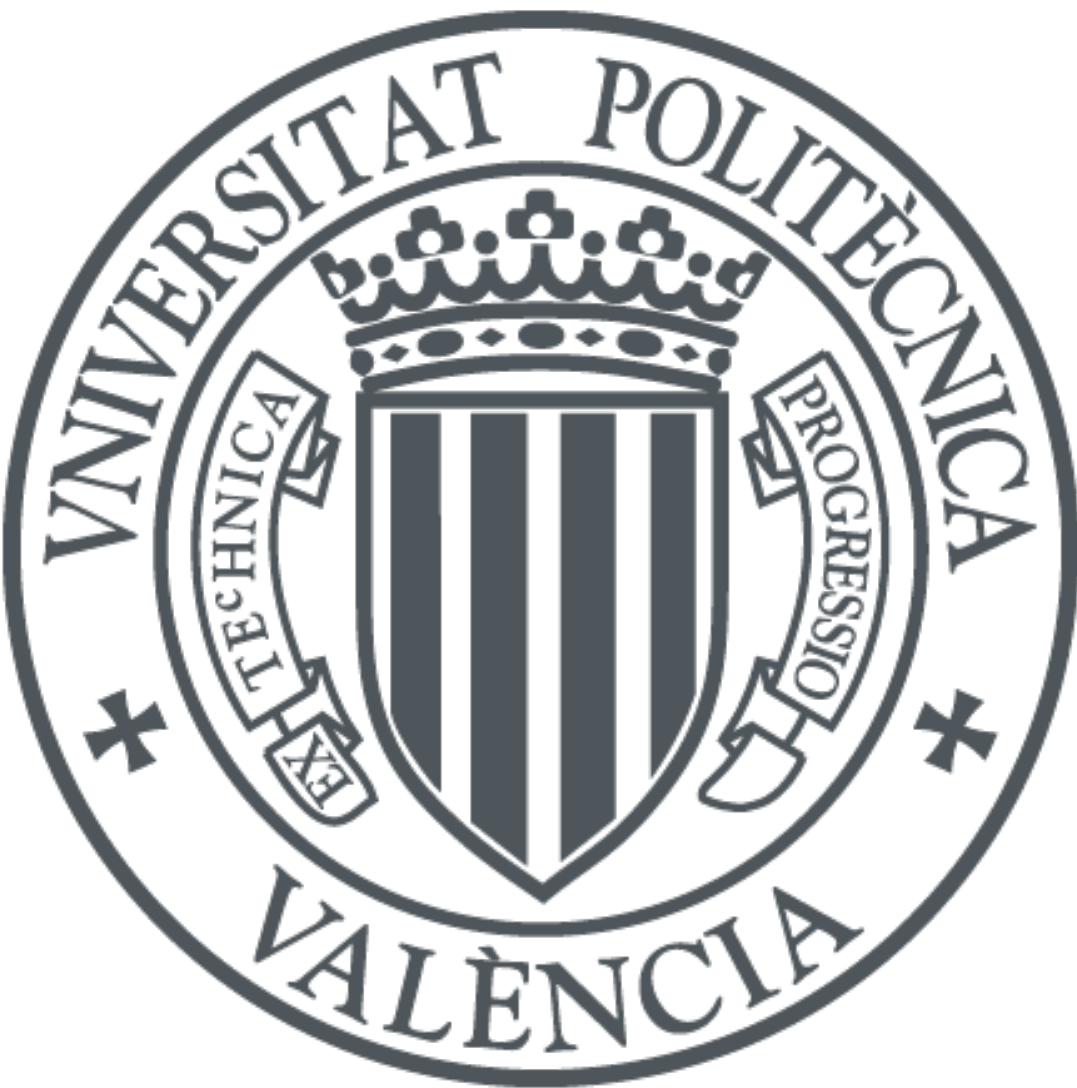

The final publication is available at

http://dx.doi.org/10.1179/003962610X12747001420825

Copyright Taylor \& Francis: STM, Behavioural Science and Public Health Titles

Additional Information

This is an author's accepted manuscript of an article published in:

"Survey Review"; Volume 43, Issue 319, 2011; copyright Taylor \& Francis; available online at: http://dx.doi.org/10.1179/003962610X12747001420825 


\title{
ON STANDARD REDUCTIONS TO RELATIVE GRAVITY MEASUREMENTS. A CASE STUDY THROUGH THE ESTABLISHMENT OF THE NEW LOCAL GRAVITY NET IN THE PROVINCE OF VALENCIA (SPAIN)
}

\author{
A. Martín, A.B. Anquela, J. Padín, J.L. Berné \\ Departamento de Ingeniería Cartográfica, Geodesia y Fotogrametría, Universidad \\ Politécnica de Valencia, C/ Camino de Vera s/n, 46520, Valencia, Spain.
}

\begin{abstract}
Standard reductions to gravity readings due to Earth tides, ocean loading and attraction, polar motion, instrumental height and air pressure variations and loading of atmospheric masses are studied in this paper from a practical point of view, that is, taking into account their numerical values and their influence on gravimetric readings and relative gravimetric observations.

The study was carried out using the observations and definition of a new local gravimetric net. This new local gravimetric net has been established in the province of Valencia (Eastern Spain) to meet the increasing requirements of geophysics, geology, geodesy and geodynamics. The net comprises 21 sites, which are an average of $45 \mathrm{~km}$ apart and was measured using Lacoste\&Romberg D203 and G301 gravimeters. Gravity values were determined using one fixed station in relation to an absolute one and 202 relative gravimetric observables. Reductions are applied for Earth tides (with real accurate amplitude and phase-difference for the principal tidal waves analysed from 301 digitally recorded days of gravity readings) where oceanic attraction and loading has been considered. In addition, reductions for polar motion, vertical gradient to instrument height and air pressure and loading of atmospheric masses have been applied. The net was established using least square adjustment where the weights of each relative gravimetric observable were determined by iterative estimation in accordance with the Huber robust estimation procedure. Obtained standard deviations of the final gravity values have an average value of $18 \times 10^{-8} \mathrm{~ms}^{-2}(18 \mu \mathrm{Gal})$, minimum value of $10 \times 10^{-8} \mathrm{~ms}^{-2}$ and maximum value of $26 \times 10^{-8} \mathrm{~ms}^{-2}$. The statistical analysis of the results concludes with a precision and reliability determination.

Discussion of the numerical values obtained in the standard gravimetric reductions shows the importance of each one in the final solution, bearing in mind that the relative gravimetric observables have been obtained using Lacoste\&Romberg instruments and the geographical location of the net. The main conclusion is that only Earth tides reduction (with approximate amplitude and phase-difference numbers for the principal tidal waves) have to be taken into account.
\end{abstract}

\section{INTRODUCTION}

Two basic objectives are the main goals of this paper: the first one is the analysis of the standard gravimetric reductions applied to gravimetric readings and relative gravimetric observables using Lacoste\&Romberg instruments; the second one is the establishment of a new local gravimetric network in the Province of Valencia.

As regards the first objective, it is a common procedure to reduce gravimetric readings due to Earth tides, ocean loading and attraction, polar motion, instrumental height and air pressure variations and loading of atmospheric masses. The procedure is adopted from absolute gravimetry, (e.g. [5]), but it is applied to relative gravity determinations too, (e.g. [2], [17], [21]). An analysis of the numerical importance of each one of these corrections is presented and discussed showing that, for relative gravimetric observables with Lacoste\&Romberg gravity meters, for the configuration of the net and for the geographic 
situation of the studied area, most of these reductions are negligible and that approximate values can be used in some cases.

As regards the second objective, disciplines such as geodesy, geophysics, geology or geodynamics are currently calling for the need to implement gravimetric nets that are sufficiently dense, precise, homogenous and reliable to achieve their increasingly demanding objectives. These gravimetric nets would eventually form part of the geodesic and geophysical infrastructure of a given region or country, along with the other nets that are intrinsic to these disciplines (planimetric or altimetric geodetic networks, seismic network, etc). This viewpoint fathered the idea of establishing a gravimetric network with these characteristics in the Province of Valencia (Eastern Spain), so users could have rapid access to a known gravity value, enabling the gravimetric datum of the Province to become a reality.

The section below is devoted to the establishment of the gravimetric net, it comprises the selection of the stations, measuring campaign, reductions to be applied to the gravimetric readings and the adjustment of the relative gravimetric observables with a brief discussion on the results obtained. The next section concerns the analysis of the applied reductions taking into account the accuracy of the gravimeters used, the configuration and geographic situation of the net. A brief conclusion ends the paper.

\section{ESTABLISHMENT OF THE GRAVIMETRIC NETWORK}

\section{Location of the vertices}

Since Valencia is a geologically stable area with low seismic activity, the vertices have been located in towns to ensure quick and easy accessibility. Most of them have been placed in churches or in town halls to ensure they were situated on rock, taking care not to locate any of them near ravines, dams, large rivers or on marshy ground, and separating the sites, as far as possible, from busy highways or main roads to avoid micro-seismicity.

After several distributions had been considered, the final distribution of the net can be seen in Figure 1, from which it can be deduced that the net will comprise 21 sites. twelve of these stations are placed in churches, five in town halls, two on railway stations, one on a chapel and one on the Polytechnic University of Valencia (U.P.V.), spaced on an average of 40-50 Km apart.

The U.P.V. vertex is the main vertex on the net, it is located at the Laboratory of Gravimetry in the Department of Cartographic Engineering, Geodesy and Photogrametry at the Polytechnic University of Valencia, with coordinates in ETRS89 reference system $\left(39^{\circ} 28\right.$ '50.9773" N, $0^{\circ} 20$ '15.536419" W, 80.608 ellipsoidal height), obtained from the GPS permanent station in the same building. The absolute gravity value drawn from the PUIG absolute gravity vertex is a known value. The PUIG vertex, pertaining to the zero order gravimetric net of Spain [16], is situated some $20 \mathrm{Km}$ to the north of the U.P.V. vertex and was observed in the year 2002 by the National Geographic Institute (I.G.N.) using the absolute gravimeter FG5\#211. The value of absolute gravity on PUIG is $980139842 \pm 3 \times 10^{-8} \mathrm{~ms}^{-2}$. 


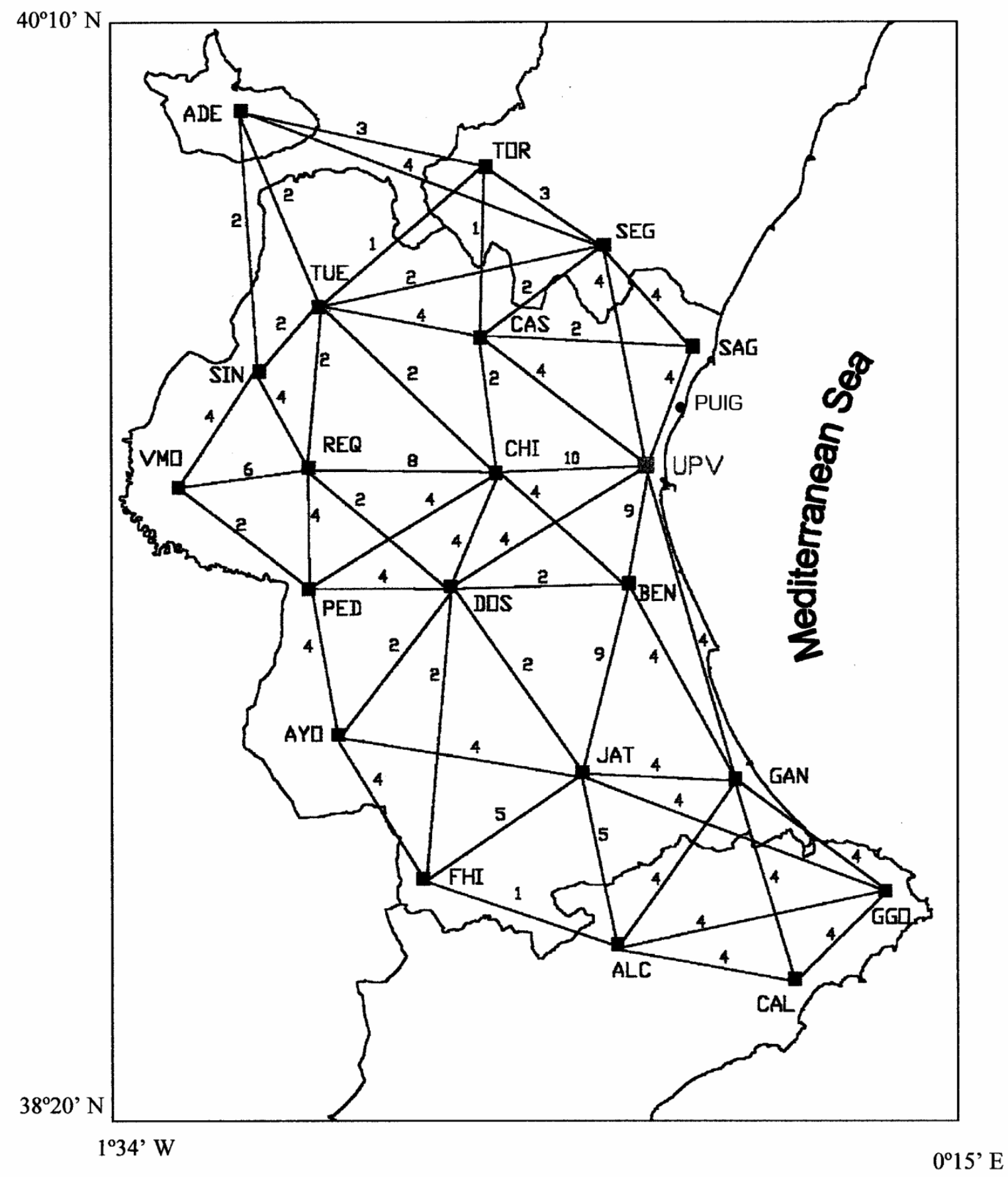

Fig. 1. Final diagram of the network observed showing the connections between the different vertices and the number of times each connection has been observed. The absolute gravity value at the U.P.V. vertex is known from the I.G.N. absolute gravity vertex PUIG.

Network readings 
The Lacoste\&Romberg D203 gravimeter equipped with an electrostatic feedback system, belonging to the Department of Cartographic Engineering, Geodesy and Photogrammetry at the Polytechnic University of Valencia, and the Lacoste\&Romberg G301 gravimeter, belonging to the I.G.N. were used to observe the gravimetric net. Several calibration lines were used to check the gravimeters and possible scale factors before they were used in the network observations, [14]. With these calibration lines the gravimeters show differences in the range of 5 to $12 \times 10^{-8} \mathrm{~ms}^{-2}$ for D203 and 3 to $10 \times 10^{-8}$ $\mathrm{ms}^{-2}$ for G301. Acceptable values taking into account the noise of the gravimetric observable as will be explained in the next section.

A total of 9 itineraries based on vertex accessibility were established and observed between January and May, 2000. The $A, B, C, D, \ldots, A$ sequence was adopted for observation purposes, exiting and closing always at the U.P.V. vertex. The itineraries have a mean total observation time of 10 hours which guarantees optimum closure of each itinerary.

202 relative gravimetric observables were measured, each site connecting with at least another three, reaching a maximum of 10 different observables for some lines of the net. Figure 1 shows all the connections established for the net and the number of times each connection has been observed.

The readings were taken rigorously following the gravimetric good practice: taking extreme care during transport, orientation towards the North, high precision instrument height reading, waiting at least 5-10 minutes before beginning the reading operations, taking the necessary readings until three valid were obtained, recording the time, atmospheric pressure and temperature at every reading.

Data were recorded whilst the two gravimeters were operating simultaneously in order to obtain better control of both the gravimeters and the itineraries, Figure 2; that is, independent determination of gravity by means of two gravimeters under the same measuring conditions was carried out.

For data recording, two barometers and one electronic thermometer were used. A digital watch, checked before and after each itinerary to ensure it was in good working order, was used to record the time.

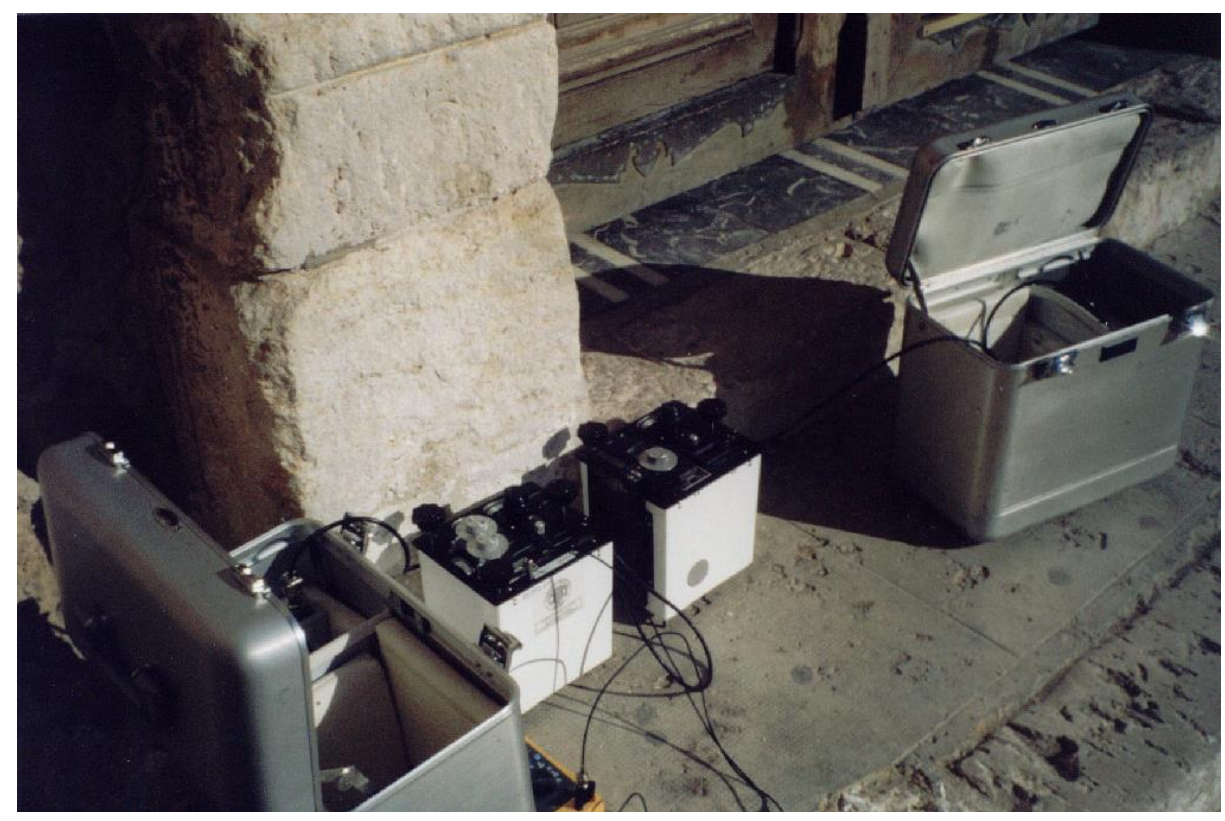


Fig. 2. An example of gravity field observations with the two gravimeters working simultaneously.

\section{Gravity readings reductions}

Gravimetric readings have been reduced for the influence of tides, polar motion, instrument height and external air pressure change in compliance with the recommendations of the International Gravity Commission, [5].

In order to predict the tide component, version 3.32 of Professor Wenzel's ETERNA program were used, since this includes the most complete tidal wave development (Hartmann-Wenzel catalogue with 12935 wave components), [24], [25].

Once the tidal potential development has been chosen, it is advisable to obtain the local real amplitude and phase-difference parameters from the principal tidal wave groups in the area of definition. These parameters will depend on the rheological nature of the working area, and must be obtained via observations.

As a first approximation, amplitude and phase-difference values for the principal tidal waves have been available in the Iberian Peninsula since 1990, [6], based on continuous observations carried out since 1974 at 19 stations evenly distributed throughout the Peninsula.

However, for a better approximation, it was decided to obtain these parameters at the U.P.V. station, and to apply them at all the stations in the Gravimetric network.

Lacoste\&Romberg D203 gravimeter was used to carry out these observations. The data (high precision gravity variations) were digitally recorded at 60 -second intervals. Commencing in February 2001 and finalising in April 2002, observations were carried out on a total of 301 days, meaning that a total of 434600 observations were processed, divided into 42 different data blocks containing from a few days to almost a month of continuous recording. A little window showing the gravity values observed, the theoretical Earth-Tidal component and the drift curve of the gravimeter can be seen in Figure 3. 


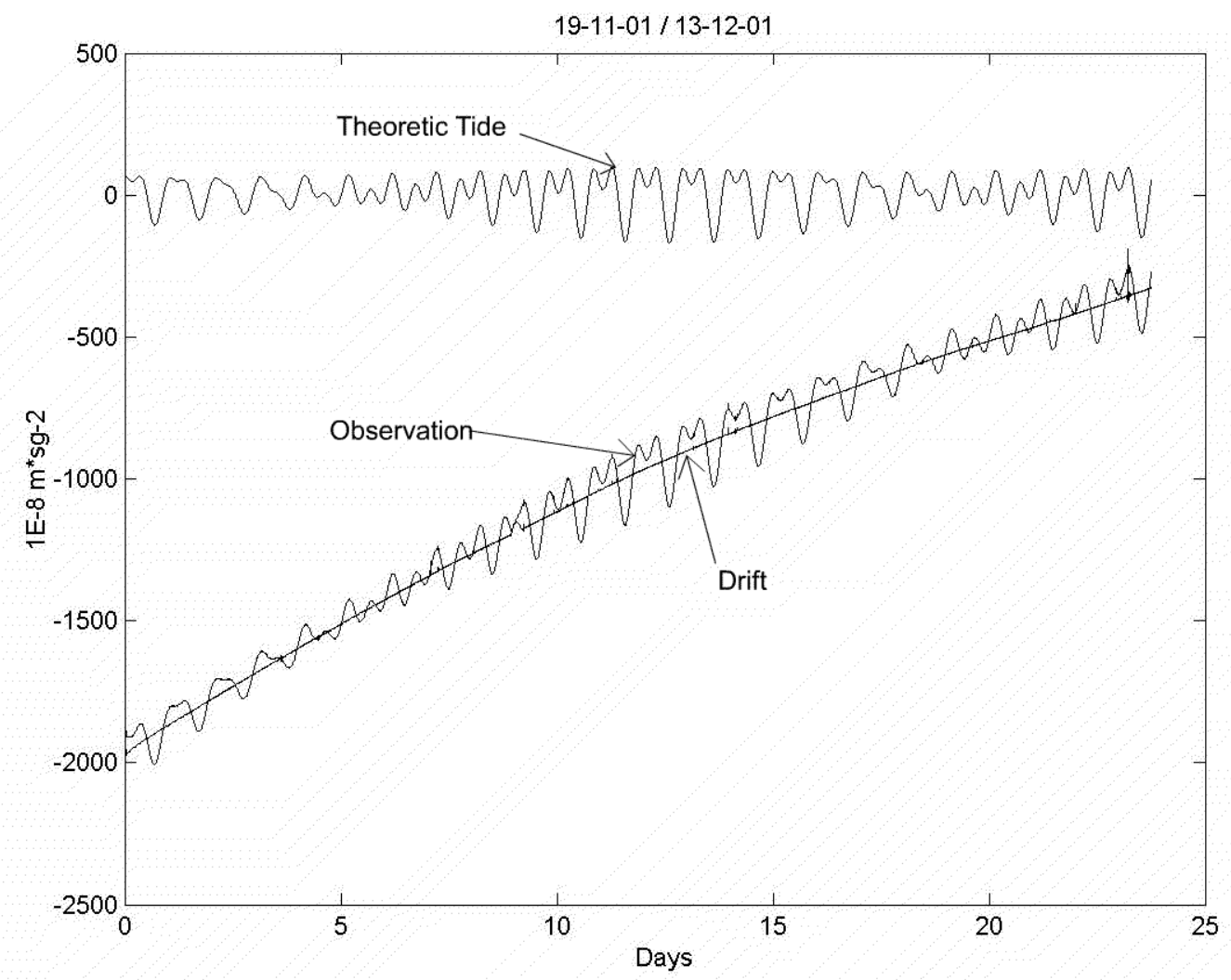

Fig. 3. Gravity values observed, theoretical tide and drift curve for the Lacoste\&Romberg D203 gravimeter at the UPV vertex between November 19 and December 13, 2001.

The data produced by the observations were edited and processed with 3.32 ETERNA Software, using the Hartmann-Wenzel catalogue. Adjustments for polar motion and length of day (L.O.D.) have not been included, since, due to the characteristics of the gravimeter used, these corrections fall within the signal noise.

Tschebyscheff polynomials of different degrees were included in the adjustment, depending on the number of days recorded in each block, to achieve approximation to the drift and to eliminate it from the calculations of amplitudes and phase-differences.

Finally, the amplitude and phase-difference parameters for wave groups Q1, O1, M1, P1, K1, J1, OO1, 2N2, N2, M2, L2, S2, K2 and M3M6 were determined, along with their standard deviation values, Table 1, [13]. These wave groups are the principal diurnal, semidiurnal and ter-diurnal groups in that tidal potential can be grouped from a spectral analysis of the tidal potential or tidal acceleration, [20]. 
Table 1. Observed Amplitude and Phase-Difference parameters for the principal wave groups in the U.P.V. vertex used for gravity tide reductions.

\begin{tabular}{cccccc}
\hline $\begin{array}{c}\text { Wave } \\
\text { group }\end{array}$ & $\begin{array}{c}\text { Frequency range in } \\
\text { cycles per day }\end{array}$ & Amplitude & $\begin{array}{c}\text { Standard } \\
\text { deviation }\end{array}$ & $\begin{array}{c}\text { Phase-difference } \\
\text { in deg. }\end{array}$ & $\begin{array}{c}\text { Standard } \\
\text { deviation }\end{array}$ \\
\hline Q1 & $0.501370-0.911390$ & 1.15171 & 0.00147 & -0.4498 & 0.0735 \\
O1 & $0.911391-0.947991$ & 1.15372 & 0.00030 & -0.4092 & 0.0150 \\
M1 & $0.947992-0.981854$ & 1.17490 & 0.00341 & -1.3241 & 0.1659 \\
P1 & $0.981855-0.998631$ & 1.15351 & 0.00067 & 0.5613 & 0.0331 \\
K1 & $0.998632-1.023622$ & 1.14420 & 0.00022 & 0.0859 & 0.0108 \\
J1 & $1.023623-1.057485$ & 1.13238 & 0.00364 & 0.1570 & 0.1834 \\
OO1 & $1.057486-1.470243$ & 1.14170 & 0.00600 & 1.1050 & 0.3012 \\
2N2 & $1.470244-1.880264$ & 1.12476 & 0.00389 & 2.1452 & 0.1979 \\
N2 & $1.880265-1.914128$ & 1.14879 & 0.00083 & 3.1514 & 0.0413 \\
M2 & $1.914129-1.950419$ & 1.16499 & 0.00017 & 2.6252 & 0.0083 \\
L2 & $1.950420-1.984282$ & 1.21062 & 0.00842 & 0.1342 & 0.3982 \\
S2 & $1.984283-2.002736$ & 1.18124 & 0.00036 & 1.5978 & 0.0175 \\
K2 & $2.002737-2.451943$ & 1.16590 & 0.00132 & 2.4225 & 0.0648 \\
M3M6 & $2.451944-7.000000$ & 1.11843 & 0.00474 & -1.8178 & 0.2425 \\
\hline
\end{tabular}

The standard deviation of the unit weight turned out to be $3 \times 10^{-8} \mathrm{~ms}^{-2}$, which validated the observations adjustment process, bearing in mind that the observations had been carried out with a Lacoste\&Romberg gravimeter.

For wave groups of less frequency and groups S1, PSI1 and PHI1, which should be determined with observations over several years, [26], the values of amplitude and phasedifference used were those obtained in the work of Camacho and Vieira, [6].

Corrections have been applied to the gravity readings of the net with respect to the direct effect on gravity measurement caused by the permanent tidal wave component, however the indirect effect of terrestrial deformation has been maintained, as the I.A.G. itself recommends, [15].

It is worth to say that amplitude and phase-difference parameters obtained for the correct prediction of the Earth tide component contain the effects of oceanic loading and tides, since the gravimetric observations to fix them have not been subjected to any filtering process in order to eliminate them. Therefore, the Earth tide corrections made to the gravimetric network data already contain these components.

The following polar motion expression was used to correct the readings, [20], [22]:

$$
\delta g_{\text {pole }}=-1.16 \omega^{2} R \sin 2 \varphi\left(x_{p}(t) \cos \lambda-y_{p}(t) \sin \lambda\right)
$$

where $\omega$ is the angular rotation of the Earth, $R$ is the Earth radius, $(\varphi, \lambda)$ are the geodetic station coordinates, and $\left(x_{p}(t), y_{p}(t)\right)$ are the coordinates of the pole position at time $t$ (the data are taken from the IERS electronic bulletin $b$, http://www.iers.org).

For the purpose of reducing the gravimetric reading to the ground $r_{i}^{c}$, the following expression was used, [19]: 


$$
r_{i}^{c}=r_{i}+\frac{\partial g_{P}}{\partial h} h_{i}
$$

where $r_{i}$ is the gravimeter reading, $\partial g_{P} / \partial h$ is the vertical gravity gradient for the measuring point (the theoretical gradient of $308.6 \times 10^{-8} \mathrm{~ms}^{-2}$ by meter was used), and $h_{\mathrm{i}}$ is the height of the instrument above the station level.

Finally, corrections were applied to the readings for the influence of atmospheric pressure changes and deformation caused by the load of atmospheric masses. This reduction has to be applied in order to account for the difference between actual atmospheric pressure and normal atmospheric pressure at the station [3], [5]. I.A.G. resolution No 9, 1983, recommends the use of the following formulae for atmospheric direct and indirect gravity reduction [19]:

$$
\begin{aligned}
& d g_{P}=0.30\left(P_{i}-P_{i n}\right) 10^{-8} \mathrm{~ms}^{-2} \\
& P_{i n}=1013.25\left(1-\frac{0.0065 H}{288.15}\right)^{5.2559}
\end{aligned}
$$

where $H$ is the orthometric height of the station, $P_{i}$ is the measured value of the pressure at the station and $P_{\text {in }}$ is the normal pressure (both in $\mathrm{hPa}$ ).

\section{Adjustment of the gravity network}

Once all the itineraries have been analysed for closure errors (based on accidental and drift function errors) and checked to ensure they fall within tolerance levels, [14], the gravimetric network can then be adjusted.

The following type of observation equation was used for each of the 202 relative gravimetric observables between the vertices $i$ and $j,[3],[4],[17],[19]$ :

$$
\left[\begin{array}{lll}
1 & -1 & -\left(Z_{j}-Z_{i}\right)-\left(t_{j}-t_{i}\right)
\end{array}\right]\left[\begin{array}{c}
g_{j} \\
g_{i} \\
\delta f_{c a l} \\
b_{l}
\end{array}\right]=\left[\left(\overline{Z_{j}}-\overline{Z_{i}}\right)\right]+v_{i, j}
$$

where $Z$ is the reading of the meters at time $t$ obtained by applying the constants obtained from the calibration tables supplied by the manufacturer, $\delta f_{c a l}$ is the variation of the calibration factor considered, a priori, as the unit (one calibration factor has to be included for every instrument used in the observation), $g$ refers to the unknown gravity values of the vertices (except for the U.P.V.), $b_{l}$ is the linear drift for the itinerary $l$, the value $\left(\overline{Z_{j}}-\overline{Z_{i}}\right)$ corresponds to the gravity readings transformed in accordance with the manufacturer's calibration table and corrected for all the effects described in preceding subsection, and, finally, $v_{i, j}$ is the residual of the observable.

The unknown values of the adjustment are, on the one hand, the gravity values for the 20 vertices that comprise the net, excluding the U.P.V. vertex that is considered a fixed 
vertex, and on the other hand, the drift parameters for each itinerary and gravimeter, that is, a total of 17 drift parameters. Unfortunately, it is impossible to determine the variation of the calibration factor, $\delta f_{c a l}$, for the two gravimeters since the absolute gravity value is only known at a single point. So these unknowns, understood to be scale factors, can not be calculated, and a zero value is assumed for expression (4). Thus the formed system of equations possesses a total of 37 unknown values.

The weight of the observables is established in accordance with the following classical expression used in a least squares adjustment procedure:

$$
P=\frac{\sigma_{O}^{2}}{\sigma_{i}^{2}}
$$

where $\sigma_{o}^{2}$ will be the variance estimator of unit weight, normally one, and $\sigma_{i}^{2}$ will be the square of the typical standard deviation of the observables, which, in this case in particular (relative gravimetric observation), will be represented by the value:

$$
\sigma_{i}=e_{a} \sqrt{2}
$$

Where $e_{a}$ is the squared component of the accidental errors at one station for each of the gravimeters. A value of $25 \times 10^{-8} \mathrm{~ms}^{-2}$ for gravimeter D203 and $30 \times 10^{-8} \mathrm{~ms}^{-2}$ for G301 were used, [19], [23].

Due to the extreme complexity involved in the gravimetric readings with regard to the precise determination of accidental errors in the reading process, which could lead to a relative gravity observation between two vertices at a given time quite different from other observations obtained at the same section at other times, so it can be expected that this weight strategy should be excessively optimistic for some observables.

To resolve this problem, robust estimation can be used. In this case, the method based on the Huber estimator was chosen, according to which the weight matrix should be redefined after the adjustment is made, based on the vector of residuals; entering, thus, into an iterative process that concludes when the results converge.

The elements of the re-weighted matrix in an $i+1$ adjustment, will be calculated by multiplying the initial values of the matrix of the previous iteration $i$ by a given factor that will be calculated according to the criteria:

$$
\begin{gathered}
\text { if }\left|v_{i j}\right| \leq k \sigma \Rightarrow \text { multiplication factor }=1 \\
\text { if }\left|v_{i j}\right|>k \sigma \Rightarrow \text { multiplication factor }=\frac{k \sigma}{\left|v_{i j}\right|}
\end{gathered}
$$

where $k$ is a given critical value that defines characteristics such as the speed of convergence of the solution (usually taking values between 1 and 3, [1]) and $\sigma$ is a given deviation value of the observables which must be fixed a priori. A value of 1.5 has been adopted for $k$, this being usual value by which the deviations are multiplied in surveying and geodesic tasks to calculate the maximum error. The deviation values $\sigma$ will be those obtained in accordance with equation (6) for each of the gravimeters used. Similar criteria 
to those used were considered for the adjustment of other gravimetric networks (e.g. [8], [12]).

Using this criterion, the net with its 202 relative gravimetric observables has been resolved; the results obtained showing that, at the third iteration, the results converge at values lower than $1 \times 10^{-8} \mathrm{~ms}^{-2}$. The results for this third iteration show an estimator of unit weight, a posteriori, of 0.93 , which passes the different statistical tests perfectly.

In Table 2 there are definite values of gravity acceleration given in IGSN71 datum for all the points of the Gravimetric Network of the Province of Valencia, as well as the accompanying standard deviations obtained in the adjustment process. UPV vertex is included in the adjustment as a known gravity vertex, with gravity value $980114362 \times 10^{-8}$ $\mathrm{ms}^{-2}$.

Table 2. Definite gravity values in the points of the Gravimetric Network of the Province of Valencia and their standard deviations. Units in $10^{-8} \mathrm{~ms}^{-2}$

\begin{tabular}{cccccc}
\hline Point & $\mathrm{g}$ & $\sigma_{\mathrm{g}}$ & Point & $\mathrm{g}$ & $\sigma_{\mathrm{g}}$ \\
\hline ADE & 979921391 & 26 & GAN & 980085433 & 24 \\
ALC & 979895915 & 17 & GGO & 980054906 & 23 \\
AYO & 979927199 & 17 & JAT & 980038285 & 15 \\
BEN & 980098235 & 12 & PED & 979926219 & 17 \\
CAL & 979995856 & 21 & REQ & 979934456 & 14 \\
CAS & 980037743 & 17 & SAG & 980136262 & 18 \\
CHI & 980035456 & 10 & SEG & 980061420 & 16 \\
DOS & 980005161 & 13 & SIN & 979897386 & 25 \\
TOR & 979903102 & 25 & TUE & 979966359 & 18 \\
FHI & 979915446 & 18 & VMO & 979925476 & 20 \\
\hline
\end{tabular}

In addition to Table 2, a statistical summary of standard deviations obtained for the final gravity values and drift parameters can be seen in Table 3.

Table 3. Statistical summary of the standard deviations obtained for the adjusted values of gravity and drift parameters

\begin{tabular}{cccc}
\hline Standard deviations & Mean & Max. & Min. \\
\hline Gravity values in $\mathrm{ms}^{-2}$ & $18 \times 10^{-8}$ & $26 \times 10^{-8}$ & $10 \times 10^{-8}$ \\
Drift parameters in $\mathrm{ms}^{-2}$ per hour & $6 \times 10^{-8}$ & $10 \times 10^{-8}$ & $4 \times 10^{-8}$ \\
\hline
\end{tabular}

The obtained accuracy shows that the relative gravimeters behaved in a stable way during the measurements series and that the measurements have been carried out systematically and carefully, which resulted in good results having in mind the accidental errors (or noise) of the instrument used as will be explained in the next section.

The rest of the statistical criteria for the final adjustment (redundancy numbers, Baarda test, internal and external reliability) have turned out to be satisfactory.

In the adjustment process D203 gravimeter showed less reliability than G301 in terms of repeated gravity observables on different days, that is, the weight of a total number of 52 observables were reduced following Huber robust estimation procedure and $80 \%$ of these 
observables belong to D203 gravimeter. However, due to the great number of observables, no systematic effects in the D203 gravity observations have been observed.

Finally REQUENA vertex (REQ in Figure 1), belongs to the old definition of the first order gravity net in the Iberian Peninsula observed in 1973, [17], obviously this gravity value has not been included in the new gravity net, but the comparison between the old $\left(979934452 \times 10^{-8} \mathrm{~ms}^{-2} \pm 11.78 \times 10^{-8} \mathrm{~ms}^{-2}\right)$ and new $\left(979934456 \times 10^{-8} \mathrm{~ms}^{-2} \pm 17 \times 10^{-8} \mathrm{~ms}^{-}\right.$ ${ }^{2}$ ) values shows a discrepancy of only $4 \times 10^{-8} \mathrm{~ms}^{-2}$, so no systematic correction between old and new nets can be expected.

\section{DISCUSSION OF THE REDUCTIONS PERFORMED}

For in-depth analysis of the reductions performed and their numerical value on the relative gravimetric observable, one must consider which instruments are used, the observable generated, the configuration of the net and the work area.

Lacoste\&Romberg relative gravimeters were used in this network, and consequently the accidental errors of a gravimetric reading can be fixed at $\pm 25 \times 10^{-8} \mathrm{~ms}^{-2}$ for a $\mathrm{D}$ model and $\pm 30 \times 10^{-8} \mathrm{~ms}^{-2}$ for a $\mathrm{G}$ model, [19], [23], so the accidental errors of a relative gravimetric observable be $25 \times \sqrt{2} \times 10^{-8} \mathrm{~ms}^{-2}$ for a $\mathrm{D}$ model and $30 \times \sqrt{2} \times 10^{-8} \mathrm{~ms}^{-2}$ for a $\mathrm{G}$ model, as seen in the above section, equation 6.

Corrections and reductions that lie above or close to this accidental error value (or noise) of the relative gravimetric observable will therefore only have numeric consequences in the final adjustment. So reductions to Earth tide, polar motion, instrument height and atmospheric pressure should be carefully analysed and numerically bounded.

\section{Reductions due to tide effects}

Regarding the reduction for terrestrial tides, obviously this must be applied and it is far greater than the accuracy of a gravimetric reading. But the effect of the choice of a tide catalogue in particular to calculate this falls far below the reading precisions as can be seen in Figures 4 and 5, which show the difference in prediction of the gravimetric terrestrial tide component using different catalogues in the U.P.V. vertex during the net observation period. Figure 4 specifically shows the differences between the Tamura catalogue, [18] and Hartmann-Wenzel catalogue; the differences are lower than $\pm 0.03 \times 10^{-}$

${ }^{8} \mathrm{~ms}^{-2}$. Figure 5 shows the differences between the Cartwright-Tayler catalogue, [7], and the Hartmann-Wenzel catalogue that are less than $\pm 0.12 \times 10^{-8} \mathrm{~ms}^{-2}$. 


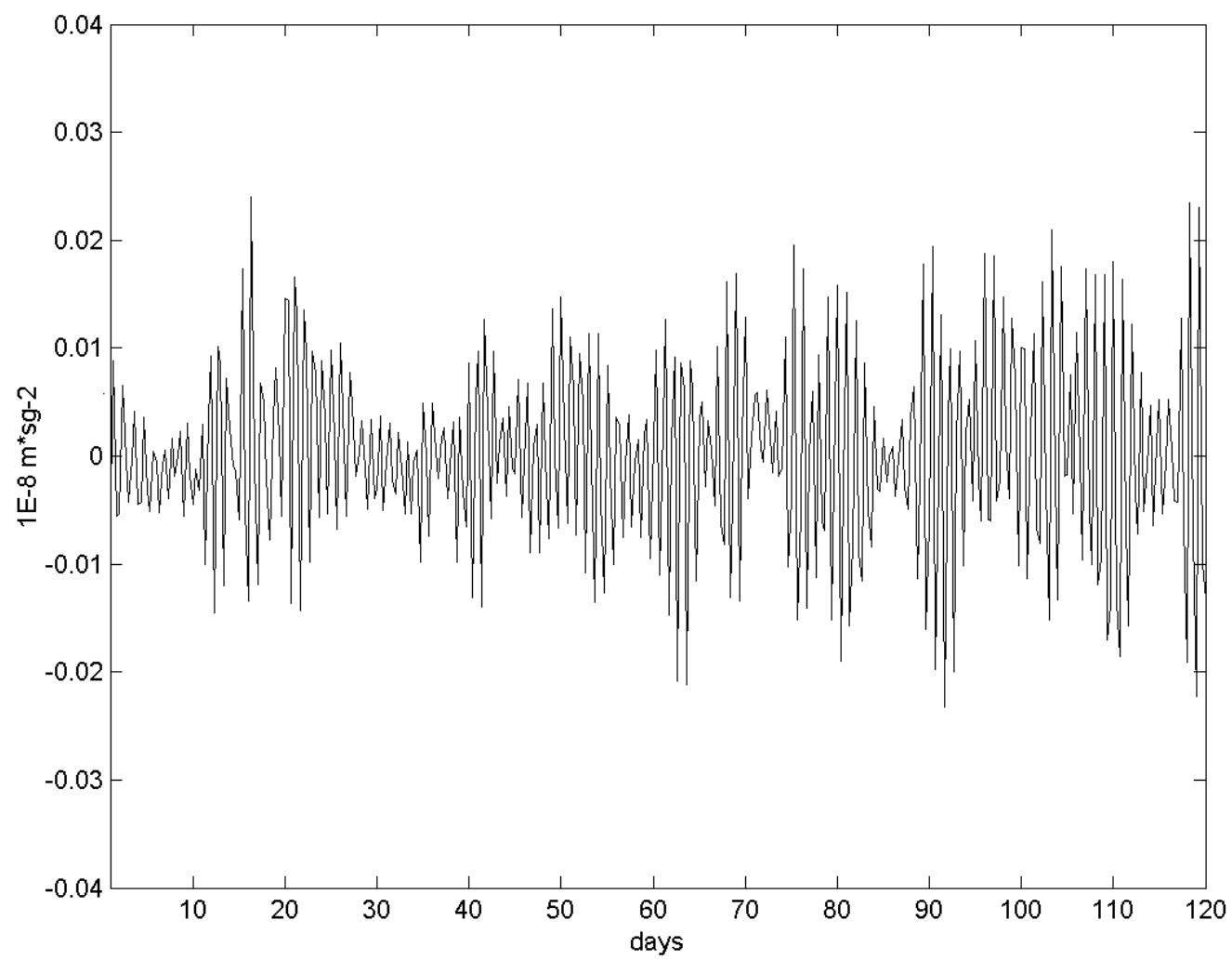

Fig. 4. Differences in the prediction of gravimetric terrestrial tides between the Tamura catalogue and the HartmannWenzel catalogue from January to May, 2000 at the UPV vertex. 


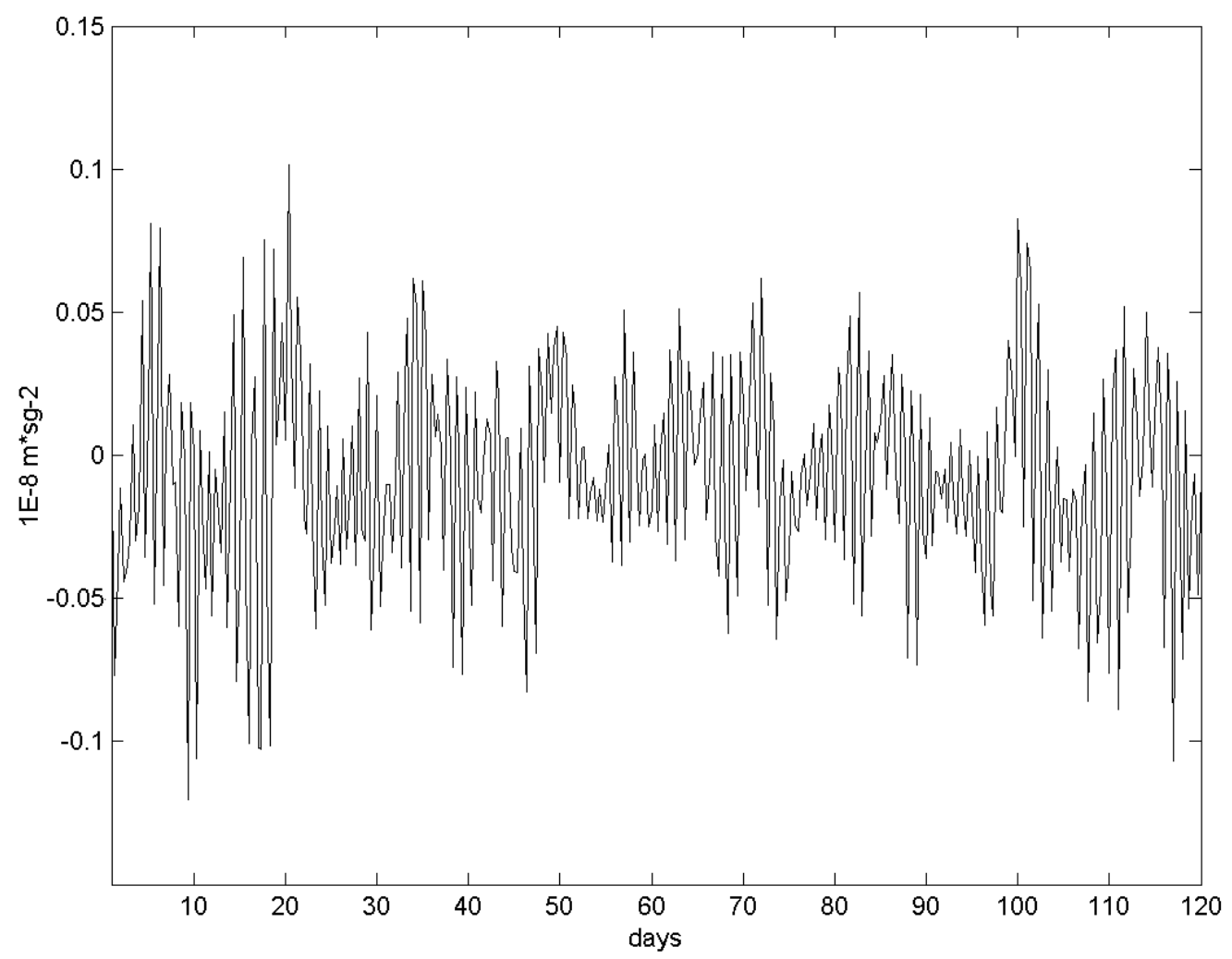

Fig. 5. Differences in the prediction of gravimetric terrestrial tides between the Cartwright-Tayler catalogue and the Hartmann-Wenzel catalogue. From January to May, 2000 at the UPV vertex.

Similarly, the fact of working with the real amplitude and phase-difference values of the main wave groups or working with approximate values does not have an important numeric effect on the readings performed. Figure 6 shows the difference, for the U.P.V. vertex and the Hartmann-Wenzel catalogue, between the prediction using the approximate values of 1.16 and 0 for the amplitude and phase-difference of the main wave groups, respectively, and using the real values as established in Table 1. It can be observed that this difference is never greater than $\pm 4 \times 10^{-8} \mathrm{~ms}^{-2}$ and consequently it is included in the noise of the actual gravimetric reading and the fact of using approximate or real values does not numerically affect the final result. 


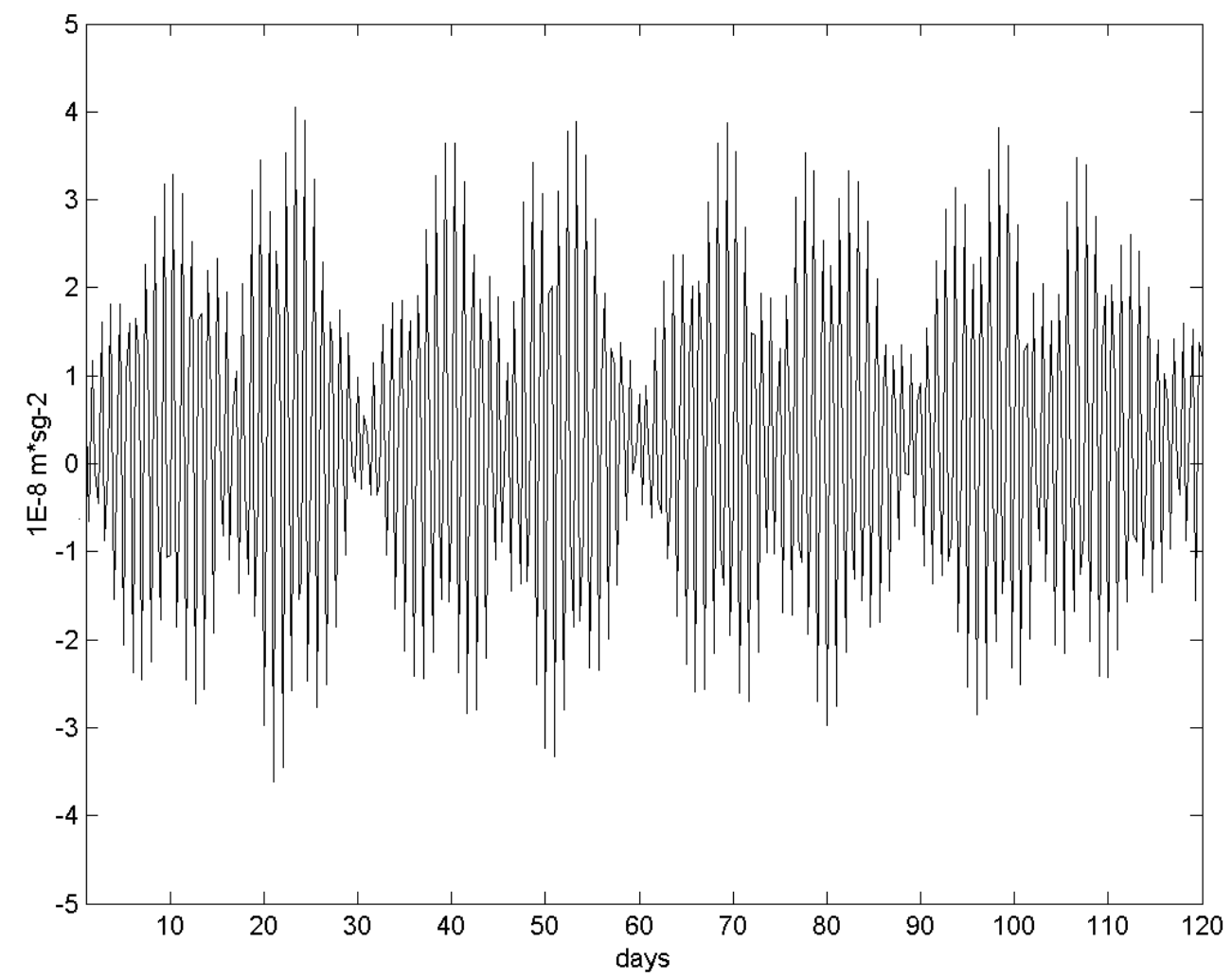

Fig. 6. Differences in the prediction of gravimetric terrestrial tides using approximate or real values for the amplitudes and phase-differences of the main tide wave groups from January to May, 2000 at the UPV vertex.

This latter conclusion can generally be reached for any vertex in the observed gravimetric network because the same instruments have always been used and consequently the same noise, and although the vertices have been observed several times to gain accuracy, these considerations in the reduction by terrestrial tides are far below the gravimetric signal obtained from the instrumental readings.

It should also be considered that the real gravimetric observable on which we will later work is relative, so these reductions to the instrumental readings will have a real effect on the gravimetric observable deducting two consecutive values. In this sense, the most important is the net configuration (the distance between vertices is located at an average of 50 kilometres) and its observation methodology (observations between vertices were carried out with a maximum two-hour time lag).

To study the effect of the choice of a specific tide catalogue on a relative gravimetric observable, the tide prediction on the U.P.V. vertex and on four hypothetical vertices located 50 kilometres to the North, South, East and West was calculated for the time period of the net observations. Secondly, deducting these four hypothetical vertices and the U.P.V. vertex were performed, taking a two-hour observation time lag, in other words, deducting the prediction of the U.P.V. vertex 2 hours earlier from the prediction of each of the hypothetical vertices. The results obtained using the Hartmann-Wenzel catalogue compared with the Tamura catalogue show a result that is never more than $\pm 0.03 \times 10^{-8}$ 
$m s^{-2}$, the same as in the study performed on a gravimetric reading. In the same way, the results obtained making a relative comparison of the Hartmann-Wenzel catalogue and Cartwright-Tayler catalogue, and also a relative comparison of the differences between using approximate amplitude and phase-difference parameters for the main tide wave groups or the real values observed, never exceed the values obtained in the comparison on a gravimetric reading. The same conclusion can therefore be reached: the use of a specific tide catalogue and the use of approximate amplitude and phase-difference values for the main tide wave groups will not numerically affect the final result of the network, because these considerations in the reduction of terrestrial tides are well below the signal of the relative gravimetric observable.

In addition, the fact of using real coefficients for these amplitudes and phase-differences that include information on oceanic load and oceanic tides but which were determined from a single station (U.P.V. vertex), means that the same values are being applied to all the net vertices. To study this fact in depth, first of all the effects of the oceanic load on all the stations should be calculated and secondly we should study the gravity effect of ocean mass variations due to the tides.

To calculate the oceanic load, the free software provided by the Center for Astrophysics and Space Science has been used. Oceanic model CSR3.0 and the Green functions on the terrestrial model of Gutenberg-Bullen, [9], which does not represent any practical difference compared with the terrestrial model PREM, were used [11]. The results obtained show that in no vertex of the net the effect of the oceanic load exceeds $\pm 0.4 \times 10^{-8}$ $\mathrm{ms}^{-2}$ during the observation period. Consequently, considering the accuracy of the readings and the gravimetric observable, we can conclude that the consideration of the oceanic load and its variation between stations can be neglected.

To calculate the effect on the gravimetric readings provoked by the movement of the oceanic masses caused by the tides, the ETERNA 3.32 software, the Hartmann-Wenzel catalogue and the real amplitude and phase-difference parameters observed at the U.P.V. station were used. The result is that the value of the sea level in the survey zone may undergo variations of up to $\pm 0.5 \mathrm{~m}$ (which matches the real tide values in the zone). These value implies variations of up to $\pm 5 \times 10^{-8} \mathrm{~ms}^{-2}$ at stations near the coast and less than $1 \times 10^{-8} \mathrm{~ms}^{-2}$ for stations more than $50 \mathrm{~km}$ away. In any case, these values are below the accuracies of the gravimetric readings. If we consider that the gravimetric observable is relative, at a distance of 50 kilometres on average and observed with a maximum two-hour time lag, we can conclude that in no event these $\pm 5 \times 10^{-8} \mathrm{~ms}^{-2}$ have been exceeded.

Lastly, consideration of the indirect effect of permanent deformation that should remain in the observations has a numeric effect of between 1.7 and $1.0 \times 10^{-8} \mathrm{~ms}^{-2}$ in the gravimetric readings performed, considering the limit values of the geographic latitude where the observed network is found. Evidently, the fact of working with relative gravimetric observables at average distances of $50 \mathrm{~km}$. means that the numeric value of this reduction is almost zero and does not have numeric consequences on the final result of the network.

Thus, considering the accuracy of the readings and that the observable on which the net will later work is relative, we can conclude that terrestrial tides should be applied, but that the choice of a specific tide catalogue is not critical for the final solution, as is the consideration of real or approximate values for the amplitudes and phase-differences of the main tide wave groups. Also, because of the net's geographic situation, corrections for 
oceanic load and for oceanic tide are negligible. Likewise, consideration of the indirect effect of permanent deformation falls entirely within the noise of the observations.

\section{Reductions due to Polar motion}

Polar motion provokes a change in the centrifugal force and consequently a change in the value of the gravity that may be fixed at an extreme maximum value of $15 \times 10^{-8} \mathrm{~ms}^{-2}$, [22]. In the case of the network in question, the calculations performed show a maximum reduction of $1.8 \times 10^{-8} \mathrm{~ms}^{-2}$ on a gravimetric reading, which is a negligible value considering the noise level of the readings. Evidently, the fact of working with relative gravimetric observables at average distances of $50 \mathrm{~km}$ practically eliminates the significance of this reduction and therefore it does not have numeric consequences for the final result of the net.

\section{Reductions due to instrument height}

Gravity is reduced to the ground considering the standard theoretical vertical gradient. This is justified because relative gravimetric observable is used and the height of the instrument does not usually vary more than $0.01-0.02 \mathrm{~m}$ from one station to another. It should however be considered that the value of the theoretical vertical gradient may vary up to $20 \%$ if the measurements are made near the ground, as in this case, [5], [10], largely depending on the topographic variations near the observation point. In the case of the observed network, we have tried to choose points where the topography around is as regular as possible, so such extreme variation percentages in the theoretical vertical gradient are not expected. However, a $10 \%$ variation in the theoretical vertical gradient implies variations of around $8 \times 10^{-8} \mathrm{~ms}^{-2}$ for a mean height of the gravimeter of $0.25 \mathrm{~m}$. But this is a negligible value within the precision (or noise) of the gravimetric readings with which we are working.

In other words, if we take the precaution of locating the observation points in zones where there is no residual effect due to the topography, it can be considered that the variation with regard to the standard theoretical value of the vertical gradient has no numeric effect on the final results because it lies within the noise of the gravimetric readings.

Consequently, if the height of one station to another does not vary more than 0.01-0.02 $\mathrm{m}$, when applying the standard vertical gradient in both stations, and considering that the gravimetric observable is relative, the reduction due to instrumental height will have no numeric effect considering the precision of the relative gravimetric observable.

Despite this latter conclusion, it is very risky to advice to not perform the height reduction if the nearby topography of a given station is not even enough, or that the instrument height between stations varies more than $0.02 \mathrm{~m}$.

\section{Reductions due to load and atmospheric pressure}

The variations in atmospheric pressure observed with regard to a normal pressure atmosphere during the works have led to a maximum reduction of $7.6 \times 10^{-8} \mathrm{~ms}^{-2}$ and a minimum reduction of $-1.1 \times 10^{-8} \mathrm{~ms}^{-2}$. Atmospheric conditions may change quickly, and consequently the use of relative gravimetric observables at an average distance of 50 
kilometres observed with maximum time lags of two-hours, is not a sufficient guarantee to conclude that the gravimetric observable shows smaller values in this reduction to those observed at each individual station. However, if we consider the range between the maximum and the minimum value observed, it can be seen that even in this extreme case, the result would fall below the precision of the relative gravimetric observable. It can therefore be concluded that this reduction had no numeric effect on the final result of the net.

\section{CONCLUSIONS}

Considered to be sufficiently dense and homogenous for geodesic, geophysical and geodynamic purposes, a new gravimetric network has been established in the Province of Valencia, with an average distance of $45 \mathrm{~km}$ between each of its 21 vertices.

Lacoste\&Romberg D203 and G301 gravimeters were used to carry out readings on nine itineraries. The observation criteria of work simultaneously using the two gravimeters in the same place has turned out to be a very useful element of control when carrying out the observations.

The readings have all been reduced for Earth tides (including, in addition, the effects of oceanic loading and sea tides), using the Hartmann \& Wenzel catalogue and real observed values for amplitude and phase-difference for the principal groups of tidal waves obtained from more than 300 days of observation and continuous recording at the U.P.V vertex with the D203 gravimeter. The indirect part of the permanent deformation of the Earth due to the effects of the tides will continue to remain in the observations, as recommended by the I.A.G.

Likewise, corrections were applied for polar motion, gravimeter height and atmospheric pressure and loading.

The calculations and adjustment of the network were carried out using 202 relative gravity observables. The weighting process for the observables based on the robust estimation offered by the Huber estimator has shown to be tremendously useful in the adjustment. Obtained standard deviations of the final gravity values have the average value of $18 \times 10^{-8} \mathrm{~ms}^{-2}$. Thus, the Gravimetric network of the Province of Valencia has finally been established.

The full station documentation can be obtained from http://www.upv.es/unigeo/gacg web page.

Finally, an extended numerical analysis over the reductions to the gravimetric readings and gravimetric observable was carried out, showing that, from a practical point of view, only Earth tide reductions with approximate values for amplitude and phase-difference for the principal groups of tidal waves have to be taken into account. That is, with the setup, procedure and instruments used in the Valencia network, the effect of real values for amplitude and phase-difference of tidal waves, indirect part of the permanent deformation of the Earth, polar motion, vertical gravity gradient and atmospheric pressure and loading have no numerical influence in the final result. Some of the conclusions about the low influence of these corrections can be extended to other gravimetric networks over the World. 


\section{ACKNOWLEDGMENTS}

The authors wish to thank to J.L. Caturla, F. García, J. M. García, M. Crespo and S. Baselga for their help and collaboration.

\section{References}

1. Baselga, S., 2003. Cálculo, compensación e interpretación de resultados en redes locales de alta precisión observadas por GPS y topografía clásica con aplicación de análisis estadístico multivariante y técnicas de estimación robusta. Ph.D. Thesis, Departamento de Ingeniería Cartográfica, Geodesia y Fotogrametría, Universidad Politécnica de Valencia. Valencia. 251 pages. In Spanish.

2. Basic, T., Markovinovic, D. and Rezo, M., 2004. Basic gravimetric network of the Republic of Croatia, IAG International Symposium, Gravity, Geoid and Space Missions, GGSM 2004, Porto, Portugal.

3. Boedecker, G. and Ritcher B., 1981. The new gravity base net 1976 of the Federal Republic of Germany (DSGN 76). Bull. Géod., 55: 250-266.

4. Boedecker, G. and Poitevin, C., 1986. Unified European gravity network UEGN status report. Bureau Gravimétrique International, Bulletin d' Information 59: 133-143.

5. Boedecker, G., 1988. International absolute gravity basestation net (IAGBN) absolute gravity observations, data processing standars and station documentation. Bureau Gravimétrique International, Bulletin d' information 63: 51-57.

6. Camacho, A.G.. and Vieira, R., 1990. Predicción de la corrección de marea en la Península Ibérica. Física de la Tierra, 2: 87-110. In Spanish.

7. Cartwright, D.E. and Tayler, R.J., 1971. New computations of the tide-generating potential. Geophysics. J. R. Astr. Soc., 23: 45-74.

8. Charles, K. and Hipkin, R.G., 1994. British precise gravity net 1993. Joint symposium of the International Gravity Comission and the International Geoid Comission, Symposium 113: 39-45, Graz, Austria. Ed. Springer-Verlag.

9. Farrell, W.E., 1972. Deformation of the Earth by surface loads. Rev. Geophys. and Space Physic., 10: 761797.

10. Haller, L.A. and Ekman, M., 1988. The fundamental gravity network of Sweden. Bureau Gravimétrique International, Bulletin d' information 63: 75-92.

11. Jentzsch, G., 1997. Earth tides and the Ocean tidal loading. in Wilhelm, H. and Zürn, W. (ed.), Tidal phenomena. Ed. Springer-Verlag, 145-171.

12. Kenyeres, A., Boedecker, G. and Francis, O., 2001. Unified European gravity reference net 2002 (UEGN2002): A status report. International Gravity and Geoid Comission, Thesalonici, Greece.

13. Martín, A., Anquela, A.B. and Padín, J., 2003. Correcciones por efecto de marea de observables geodésicos y gravimétricos de alta precisión. Topografía y Cartografía 118-119: 56-64. In Spanish.

14. Martín, A. and Padín, J., 2004. Establecimiento de la Red Gravimétrica de la Provincia de Valencia. Publicaciones de la Universidad Politécnica de Valencia, nº. 4004, Ed. Universidad Politécnica de Valencia, Valencia. 115 pages. In Spanish. 
15. Rapp, R.H., 1983. Tidal gravity computations based on recommendations of the standard Earth Tide commitee. Bulletin d' Informations Marees Terrestres 89: 5814-2819.

16. Rodríguez, E., Fernández, M. and Núñez, E., 2003. Red gravimétrica de orden cero y primer orden en España. Proceedings of V Setmana Geomàtica de Barcelona. Barcelona. In Spanish.

17. Sevilla, M.J., Gil, A.J. and Romero, P., 1990. Adjustment of the first order gravity net in the Iberian Peninsula. Bureau Gravimétrique International, Bulletin d' information 66: 21-54.

18. Tamura, Y., 1987. A harmonic development of the tide generating potential. Bulletin d' informations des marees terrestres, 99: 6813-6855.

19. Torge, W., 1989. Gravimetry. Ed. Walter de Gruyter, Berlin-New York. 465 pages.

20. Torge, W., 2001. Geodesy. Ed. Walter de Gruyter, Berlin-New York, $3^{\text {rd }}$ edition. 416 pages.

21. Vieira, R., Camacho, A.G. and Ortíz, E., 2002. Global adjustment for the gravity calibration line MadridValle de los Caidos. Física de la Tierra, 14: 127-159.

22. Wahr, J.M., 1985. Deformation induced by polar motion. JGR, vol. 90, B11: 9363-9368.

23. Wang, Z.C. and Jiang, Z.W., 1996. Microgravimetry. Ed Science Press, Beijing, China. 146 pages.

24. Wenzel, G., 1998. Format and structure for the exchange of high precision tidal data, http://www.ife.unihannover.de/ Wenzel/format/format.html, acceded on February 1999.

25. Wenzel, G., 1998. The nanogal software: Earth tide data processing package Eterna 3.3, http://www.ife.uni-hannover.de/ Wenzel/eterna33/eterna33.html, acceded on February 1999.

26. Wenzel, G., 1999. Earth tide data processing package, Eterna version 3.32. User manual. 75 pages. 